\title{
Glycine assisted synthesis of nickel ferrite nanoparticles by auto-combustion method using microwave radiation
}

\author{
Azadeh Tadjarodi*, Sanaz Chamani \\ Research Laboratory of Inorganic Materials Synthesis, Department of Chemistry, \\ Iran University of Science and Technology \\ (E-mail: tajarodi@iust.ac.ir)
}

\begin{abstract}
Abestract
We synthesized magnetic nickel ferrite nanoparticles by auto-combustion method using microwave radiation. Iron (III) nitrate nonahydrate, nickel nitrate pentahydrate and glycine as reactants were mixed. Then, this mixture was transferred into a domestic microwave oven with a power of $900 \mathrm{~W}$ for $30 \mathrm{~min}$. The product was characterized by X-ray Diffraction (XRD), Fourier Transform Infrared spectroscopy (FT-IR) and Scanning Electron Microscopy (SEM). Also, application of synthesized nickel ferrite nanoparticles was studied for adsorption of Congo Red as an organic pollutant from aqueous solution.
\end{abstract}

Keywords: Microwave, Glycine, Nickel ferrite, Nanoparticles, Organic pigment

\section{Introduction}

Magnetic nanoparticles have taken more attention in recent years due to their special properties such as high surface area, easy separation from reaction media. Magnetic nickel ferrite nanoparticles are kind of soft magnetic ferrites. These nanoparticles have inverse spinel structure [9]. This nanomaterial is used in various fields such as electronic, electronic devices and catalysis. There are several chemical methods for the synthesis of nickel ferrite nanoparticles [16], such as sol-gel method [1,2], sol-gel auto-combustion method [5], hydrothermal method [3, 6] and co-precipitation method [4]. Amongst reported synthesis methods, the microwave method has gathered most attention due to some advantages such as feasibility, low-cost, environmental friendly and short reaction time for the synthesis of nickel ferrite nanoparticles [7]. However, 
few studies have been reported about the synthesis of magnetic ferrite nanomaterials through this method. In this study, we synthesized magnetic nickel ferrite nanoparticles using facile and fast microwave radiation method and used as an adsorbent for adsorption of congored as an organic pigment from aqueous solution.

\section{Experimental}

All chemicals were prepared from Merck Co and used without further purification.

In this work, $\mathrm{Fe}\left(\mathrm{NO}_{3}\right)_{3} .9 \mathrm{H}_{2} \mathrm{O}$ and $\mathrm{Ni}\left(\mathrm{NO}_{3}\right)_{2} .5 \mathrm{H}_{2} \mathrm{O}$ as metal sources, $\mathrm{NH}_{2} \mathrm{CH}_{2} \mathrm{COOH}$ as a fuel and organic driving agent and $\mathrm{NH}_{4} \mathrm{NO}_{3}$ as an oxidizer were used. These materials were mixed and transferred into a domestic microwave oven with a power of $900 \mathrm{~W}$ for $30 \mathrm{~min}$. The resulting compound was collected, washed several times with ethanol and distilled water for removal the residual initial materials, dried at $70{ }^{\circ} \mathrm{C}$ for $24 \mathrm{~h}$. Finally the dark brown color nickel ferrite powder is obtained. To characterize the products, different techniques of FT-IR, XRD and SEM were used.

\section{Characterization}

Fourier transform infrared (FT-IR) spectra were recorded on a Shimadzu-8400S spectrometer in the range of $400-4000 \mathrm{~cm}^{-1}$ using $\mathrm{KBr}$ pellets. The X-ray diffraction (XRD) patterns were recorded by a STOE powder diffraction system using $\mathrm{CuK} \alpha$ radiation (wavelength, $\lambda=1.54060$ $\AA$ ). Scanning electron microscopy (SEM) images were taken on VEGA $\$ TESCAN S360 with gold coating. The UV-Vis absorption study was performed at room temperature in the wavelength range of the 190-800 nm on a UV-Vis spectrometer (ShimadzuUV-1700).

\section{Adsorption experiment}

The study of congored pigment absorption in the presence of nickel ferrite nanoparticles as an adsorbent was studied at room temperature with these conditions:0.025g of nickel ferrite nanoparticles were added to the congored aqueous solution with an initial concentration in the range of 10 to $500 \mathrm{mg} \cdot \mathrm{L}^{-1}$. The resulting mixture was agitated for $3 \mathrm{~h}$ in the dark. After that, the mixture was centrifuged and the concentration of the residual pigment was measured by using UV-Vis spectrophotometer at $498 \mathrm{~nm}[8]$. 


\section{Results and Discussion}

Figure 1 shows the FT-IR spectrum of nickel ferrite nanoparticlesprepared by microwave radiation. According to Figure 1, the appeared peaks at 422 and $580 \mathrm{~cm}^{-1}$ belong to Ni-O and Fe$\mathrm{O}$ bands, respectively. There are no additional adsorption bands of the organic groups on this spectrum.

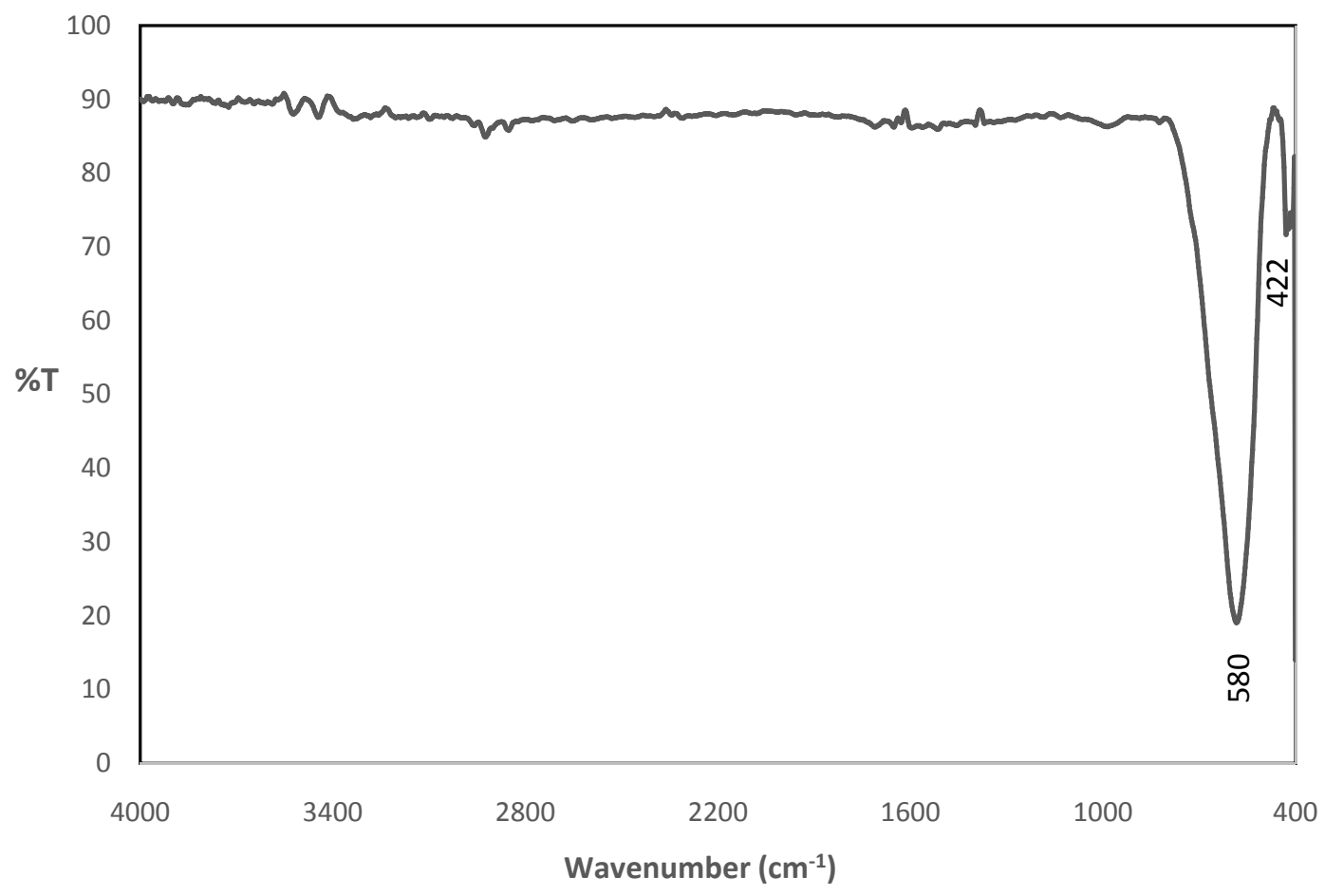

Figure 1. FT-IR spectrum of the nickel ferrite nanoparticles

The XRD pattern shows that the synthesized product is a good match with the crystalline system of cubic phase related to CAF card No. 01-088-0380 (Figure 2). The diffraction peaks at $2 \Theta$ valueof $18.44^{\circ}, 30.33^{\circ}, 35.72^{\circ}, 37.36^{\circ}, 43.39^{\circ}, 53.85^{\circ}, 57.40^{\circ}, 63.03^{\circ}, 71.54^{\circ}, 74.62^{\circ}$ and $75.59^{\circ}$ are matching with the $111,220,311,222,400,422,511,440,620,533$ and 622 planes of cubic phase with the space group of Fd-3m related to the synthesized product. According to XRD pattern, pure phase of nickel ferrite was synthesized and impurity peaks were not observed in the pattern. 


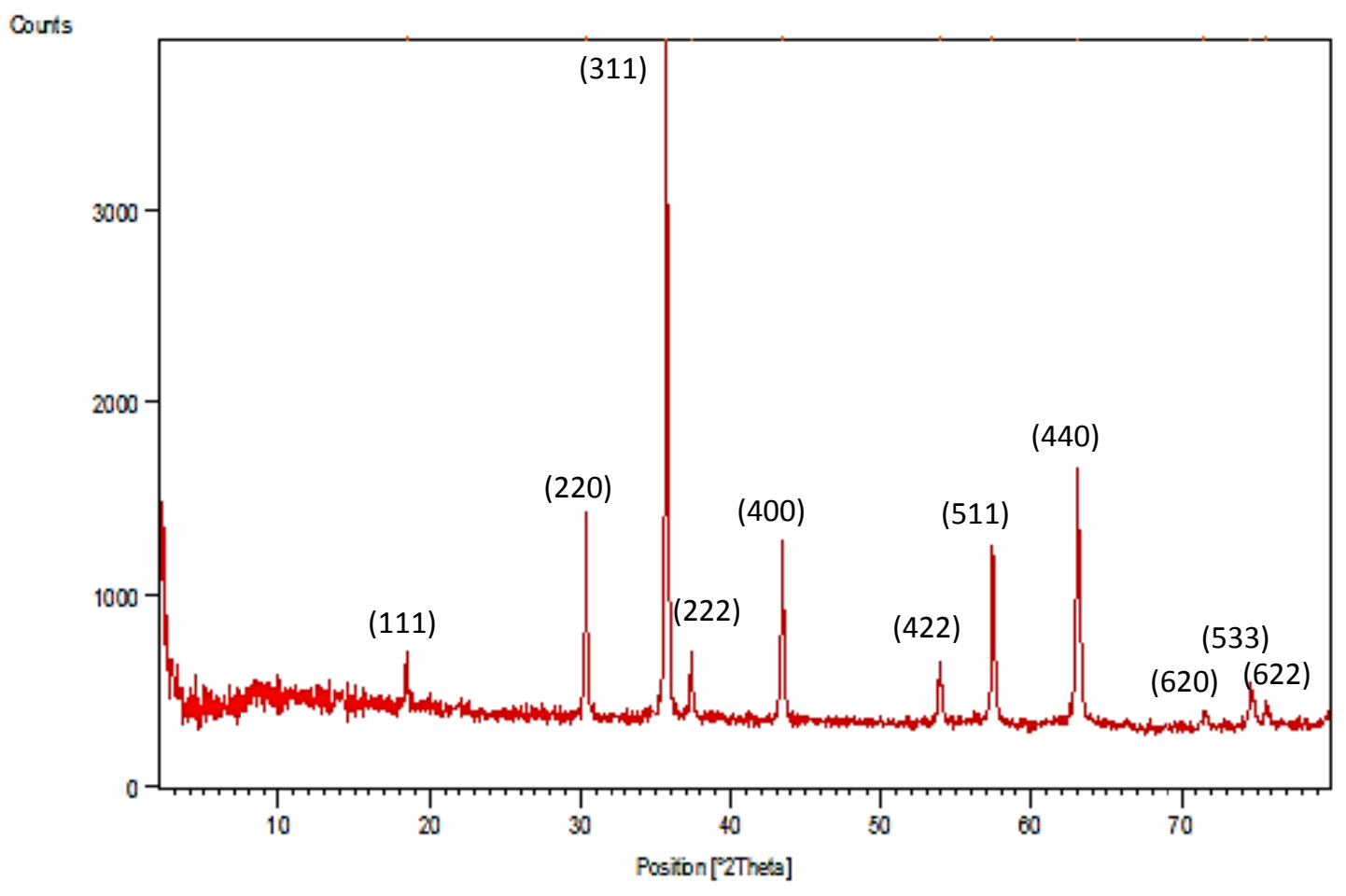

Figure 2. XRD pattern of the synthesized nickel ferrite nanoparticles.

The morphology of product was studied with scanning electron microscopy (SEM). Figure3 indicates SEM images of the synthesized nickel ferrite nanoparticles. It seems that agglomeration of particles due to high magnetism of the synthesized nickel ferrite nanoparticles.

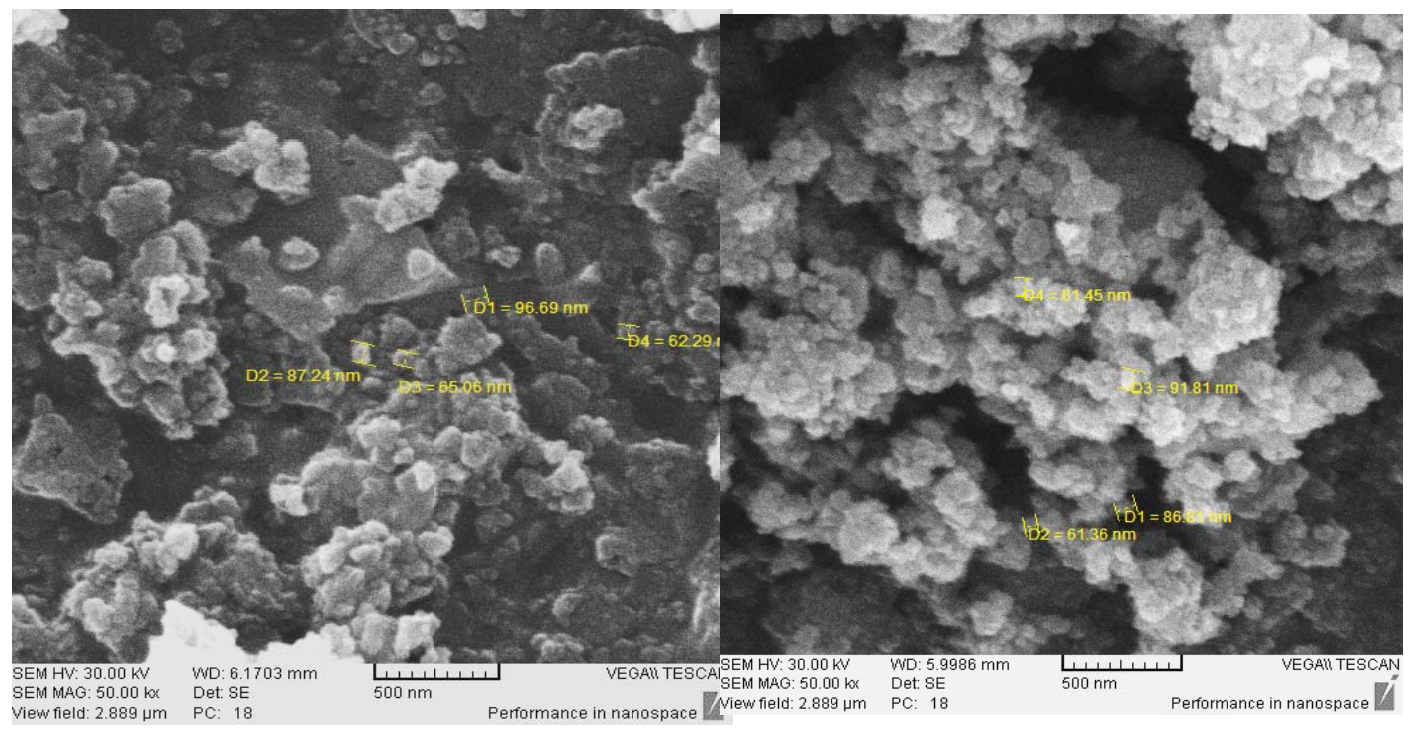

Figure 3. SEM images of nickel ferrite nanoparticles. 
The ability of nickel ferrite nanoparticles as an adsorbent to absorb congored pigment was studied. The adsorption isotherms were calculated by Langmuir and Freundlich models. The following equations are related to Freundlich and Langmuir models.

$\log q_{e}=\log K_{F}+\left(\frac{1}{n}\right) \log c_{e}$

$\frac{\mathrm{C}_{\mathrm{e}}}{\mathrm{q}_{\mathrm{e}}}=\mathrm{c}_{\mathrm{e}}\left(\frac{\mathrm{a}_{\mathrm{L}}}{\mathrm{K}_{\mathrm{L}}}\right)+\left(\frac{1}{\mathrm{~K}_{\mathrm{L}}}\right)$

The Langmuir and Freundlich models are attributed to monolayer and multilayer adsorption, respectively. Where, $K_{F}$ and $n$ are the Freundlich constants with $K_{F}\left(\mathrm{mg}^{1-1 / n} L^{1 / n} g^{-1}\right)$ is the adsorption capacity of the sorbent (Figure.4a). Also $\mathrm{a}_{\mathrm{L}}\left(\mathrm{Lmg}^{-1}\right)$ and $\mathrm{K}_{\mathrm{L}}\left(\mathrm{Lg}^{-1}\right)$ are the Langmuir adsorption isotherm constants (Figure.4b). $c_{\mathrm{e}}$ and $\mathrm{q}_{\mathrm{e}}$ in two reactions are the equilibrium concentration of congored pigment in solution $\left(\mathrm{mg} \mathrm{L}^{-1}\right)$ and the amount of adsorbed pigment molecules $\left(\mathrm{mg} \mathrm{g}^{-1}\right)$ per unit of adsorbent at equilibrium $\left(\mathrm{mg} \mathrm{g}^{-1}\right)$, respectively. Figure 4 shows the adsorption process follows the Langmuir model ( $R_{2}>0.98$ with $99 \%$ confidence level).

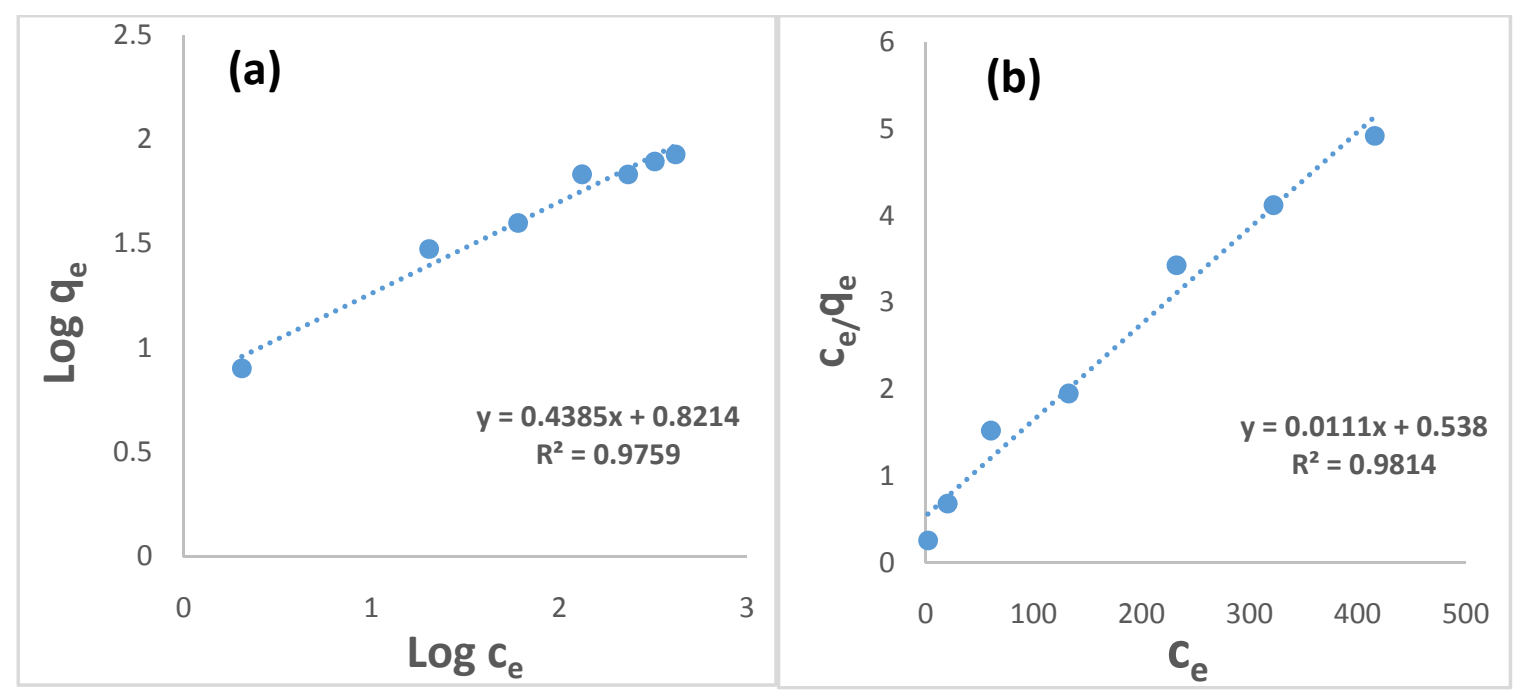

Figure4: adsorption isotherms of nickel ferrite nanoparticles as an adsorbent for absorb congored pigment, a): Freundlich isotherm, b): Langmuir isotherm

\section{Conclusions}

In summary, we used a fast, easy and environmental friendly microwave radiation method for synthesis of nickel ferrite nanoparticles. The results showed that microwave method is a suitable 
method for synthesis of magnetic nickel ferrite nanoparticles and these nanoparticles can be used as a qualified adsorbent for adsorption of congored pigment from aqueous solution. It was concluded that the adsorption process follows of the Langmuir model.

\section{References}

[1] D. H. Chen, X. R. He,"Synthesis of nickel ferrite nanoparticles by sol-gel method," Materials Research Bulletin, 36 (2001) 1369-1377.

[2] R. Sena, P. Jainb, "Synthesis and Characterization of nickel ferrite $\left(\mathrm{NiFe}_{2} \mathrm{O}_{4}\right)$ nanoparticles prepared by sol- gel method," Materials Today: Proceedings, 2 (2015) 3750 -3757.

[3] M. Srivastava, S. Chaubey, A. K. Ojha, "Investigation on size dependent structural and magnetic behavior of nickel ferrite nanoparticles prepared by sol-gel and hydrothermal methods," Materials Chemistry and Physics, 118 (2009) 174-180.

[4] K. Maaz, S. Karim, A. Mumtaz, S. K. Hasanain, J. Liu, J. L. Duan, "Synthesis and magnetic characterization of nickel ferrite nanoparticles prepared by co-precipitation route ,"Journal of Magnetism and Magnetic Materials, 321 (2009) 1838-1842.

[5] P. Sivakumar, R. Ramesh, A. Ramanand, S. Ponnusamy, C. Muthamizhchelvan, "Preparation and properties of nickel ferrite $\left(\mathrm{NiFe}_{2} \mathrm{O}_{4}\right)$ nanoparticles via sol-gel auto-combustion method," Materials Research Bulletin, 46 (2011) 2204-2207.

[6] D. Chen, D. Chen, X. Jiao, Y. Zhao, "Hydrothermal synthesis and characterization of octahedral nickel ferrite particles," Powder Technology, 133 (2003) 247 - 250.

[7] S. Komarneni, "Microwave-Hydrothermal Synthesis of Nanophase Ferrites," Journal of the American Ceramic Society, 81 (1998) 43-3041.

[8] A. Afkhami, R. Moosavi, "Adsorptive removal of Congo red, a carcinogenic textile dye, from aqueous solutions by maghemite nanoparticles," Journal of Hazardous Materials, 174 (2010) 398403.

[9] M. Aliahmad, and M. Noori, "Synthesis and characterization of nickel ferrite nanoparticles by chemical method," Indian Journal of Physics, 87 (2013) 431-434. 